\title{
Current scenario of malaria vaccine
}

\author{
Jarnail Singh Braich ${ }^{*}$, Surinder Singh Malik
}

Department of Pharmacology, Pt. BD Sharma PGIMS, Rohtak124001, India

Received: 11 September 2012 Accepted: 18 September 2012

\section{*Correspondence to:}

Dr. Jarnail Singh Braich,

E-mail: drjarnail@gmail.com

\begin{abstract}
Malaria is one of the deadliest infectious diseases that affects millions of people worldwide including India. As an addition to chemoprophylaxis and other antimalarial interventions malaria vaccine is under extensive research since decades. The vaccine development is more difficult to predict than drug development and presents a unique challenge as already there has been no vaccine effective against a parasite. Effective malaria vaccine could help eliminate and eradicate malaria; there are currently 63 vaccine candidates, 41 in preclinical and clinical stages of development. Vaccines are being designed to target pre-erythrocytic stages, erythrocytic stage or the sexual stages of Plasmodium taken up by a feeding mosquito, or the multiple stages. Two vaccines in preclinical and clinical development target P. falciparum; and the most advanced candidate is the pre-erythrocytic vaccine RTS,S which is in phase-III clinical trials. It is likely that world's first malaria vaccine will be available by 2015 at the country level. More efficacious second generation malaria vaccines are on the way to development. Safety, efficacy, cost and provision of the vaccine to all communities are major concerns in malaria vaccine issue.
\end{abstract}

Keywords: Malaria-vaccine, pre-erythrocytic (PE), blood-stage (BS), circumsporozoite protein (CSP), merozoite surface protein (MSP)

\section{INTRODUCTION}

A protozoal infection, malaria, takes lives of more than one million people every year and causes 300-500 million people to fall ill all over the world. Approximately $80 \%$ of the malaria deaths take place in Africa and $66 \%$ of the population is thought to be at risk. The majority of people who die of malaria are children less than five years of age. ${ }^{1}$ In Asia deaths due to malaria are less than $15 \%$ of global total despite the fact that $49 \%$ of the people in Asia are living under threat from malaria. In India 2 million confirmed malaria cases and 1000 deaths are reported yearly and Orissa, Madhya Pradesh, West Bengal, Chhattisgarh and Jharkhand are five most affected states. ${ }^{2}$ With the development of resistance to chloroquine and dichlorodiphenyltrichloroethane (DDT) malaria has reemerged in many parts of the world and burden of disease and death has increased significantly in malaria endemic countries with spread to new areas. ${ }^{3}$ Malaria related mortality has not decreased, rather has increased, in the past decade. ${ }^{1}$ Global burden of malaria is estimated in terms of disability-adjusted life years (DALYs) by WHO; and in Africa it is fourth leading cause of years of productive life lost to mortality and morbidity. ${ }^{4}$ Currently malaria intervention includes insecticides, insecticide treated nets (ITN), intermittent preventive treatment to pregnant women (IPTp), and artemisinin based combination therapy (ACT). ${ }^{2}$ International attention is focused on developing vaccines that can prevent malaria; thus scientists from the public and private sectors are being encouraged to accelerate malaria vaccine development.

\section{MALARIA VACCINE}

Vaccines being safe and reliable, have historically offered a highly effective and cost-effective means of preventing disease and death. Vaccines hold particular promise for malaria, and recent technological advances and evidence demonstrates that immunizing children against the malaria parasite is feasible. Development of malaria vaccine poses scientific challenges i.e. great complexity of the parasite regarding antigens, limited and incomplete knowledge about acquired immunity developed against malaria, no appropriate animal model for malaria, and high cost of developing a malaria vaccine candidate before it can be marketed. ${ }^{5-6}$ Feasibility of a malaria vaccine is justified by partial and passive immunity against most severe form of malaria. ${ }^{7}$ In 1970 nonimmune volunteers were extensively exposed to UVirradiated sporozoites and on re-challenge to normal 
sporozoites $90 \%$ of them had acquired complete but short lived immunity. ${ }^{8}$ Efficacy of experimental malaria candidate vaccine has been studied in adults and children in phase-II trials. ${ }^{9-10}$

Perfect malaria vaccine would be the one which is easy to deliver, cheap, and provides lifelong immunity with $100 \%$ efficacy against morbidity and mortality in people of all ages; and would be rapidly delivered to all countries, especially the poorest, to eradicate malaria. The reality however, is that any malaria vaccine is unlikely to be perfect and the decision to introduce the actual vaccine will pose policy challenges. ${ }^{1}$

Researchers have begun speculating about what might constitute a minimally acceptable vaccine for the developing world. The proposed efficacy levels for costeffective malaria vaccines range from low to high. ${ }^{4}$ Engers and Godal estimate that a hypothetical malaria vaccine that could be introduced into the Expanded Program on Immunization (EPI) with $30 \%$ or higher efficacy against child mortality and a duration of immunity of three years or more would be highly costeffective. $^{11}$ Once a malaria vaccine is available, policymakers will need to consider its efficacy, cost, dosing schedules and the cost of other interventions before deciding to introduce it. ${ }^{1}$

Malaria drug development is currently a research priority. Vaccine development is only one aspect of efforts to control malaria but an effective vaccine should transform prospects for reducing the burden of this disease. Preventive malaria vaccine is under development since decades and the WHO initiative for vaccine research (IVR) have made collective efforts to explore and accept the challenges. There are three intermediate goals of vaccine research: induction of strong, strain-transcending, durable immune responses; identification of protective antigens for stage-specific immunity; and successful combination of candidate immunogens. ${ }^{12}$ Program for appropriate technology in health (PATH) malaria vaccine initiative (MVI) research and development strategy is an approach to develop and licence $1^{\text {st }}$-generation malaria vaccine by 2015 having a protective efficacy of more than $50 \%$ against severe disease and death and lasts longer than one year; and next-gen vaccine by 2025 with $80 \%$ efficacy against clinical disease for 4 years- thus a landmark of 2015 for a $1^{\text {st }}$-generation vaccine and 2025 for a $2^{\text {nd }}$-generation vaccine. ${ }^{13}$

\section{NATURAL AND VACCINE INDUCED IMMUNITY AGAINST MALARIA}

Natural exposure of man to P. falciparum shows gradual short-lived strain specific malaria immunity; ${ }^{14}$ and repeated infections are required to maintain immunity which is both antibody and T-cell mediated. ${ }^{15-17}$ The aim with most vaccines is to induce antibody and T-cell responses to one or a few antigens but of greater magnitude, duration, and strain-transcendence than in naturally acquired immunity. Antigenic variation occurs in some important blood-stage malaria antigens and there is a possibility that vaccination could select for escape mutants, but this is of less a concern in malaria than with viruses such as HIV-1. T-cell responses have been neglected in blood-stage (BS) vaccine development and alternative long-term approach is to use a cocktail of many antigens to attempt to mimic natural immunity. ${ }^{18}$

\section{PRINCIPAL TARGETS OF MALARIA VACCINE} (Table 1):

- Pre-erythrocytic (PE) vaccine approaches that target $\mathrm{P}$. falciparum.

- Approaches that target P. vivax.

- Transmission-blocking vaccine (TBVs) approaches that target $\mathrm{P}$. falciparum and $\mathrm{P}$. vivax.

- Blood-stage (BS) approaches limited to those related to combination $\mathrm{P}$. falciparum $/ \mathrm{P}$. vivax transmission-blocking vaccines. ${ }^{19}$

\section{PRE-ERYTHROCYTIC (PE) VACCINES}

PE vaccines would induce high titers of functional antibodies against sporozoites to prevent all parasites entering the liver stage, and induce potent cytotoxic Tlymphocyte immunogenicity against the liver stage to kill infected hepatocytes, while not harming the human host. RTS, S-a recombinant protein vaccine is lead candidate in this category. ${ }^{20}$ Hepatitis B surface antigen DNA was fused to DNA encoding a large part of pre-erythrocytic malaria antigen, the circumsporozoite protein (CSP). ${ }^{21-22}$ The central repeat $(\mathrm{R})$ region of CSP fused to $\mathrm{C}$-terminal region known to contain T-cell epitopes (hence " $T$ ") fused in turn to the hepatitis B surface antigen $(\mathrm{S})$ yields a yeast-expressed protein RTS. The fusion product (RTS) binds hepatitis surface antigen (S) to form RTS,S particles which are mixed with a GSK adjuvant, AS02 which is a mixture of 3-O-desacyl-4-monophosphoryl lipid A (MPL), Quillaja saponaria 21 (QS21) and an emulsion and given intramuscularly on two to three occasions. RTS,S vaccination induces high titer antibodies to CSP and to $\mathrm{S}$ and gives $30-60 \%$ protection against parasites of the same strain in a sporozoite challenge model. ${ }^{20,23}$ RTS,S was effective for at least 18 months in reducing clinical malaria episodes by $35 \%$ and severe malaria by $49 \%{ }^{9}$

Next-generation PE vaccine candidates fall into one of four categories i.e.

1. Circumsporozoite protein (CSP) based PE vaccines (RTS,S/AS02, ICC-1132): ICC-1132, a hepatitis B core particle, is genetically engineered to include a region of CSP for high titer of antibody induction. ${ }^{24}$ 
2. Multi-antigen, PE-stage vaccines incorporating CSP: Sporozoites can be neutralized by circulating antisporozoite antibodies or targeted post-hepatocyte invasion by cell-mediated mechanisms. Only a single antigen, CSP, has been shown to provide high levels of protective efficacy in both controlled human challenge studies and field studies in Africa. Multi-antigen vaccines, by additional sporozoite and liver stage antigens, can complement role of anti-CSP immunity by priming additional antibodies to block hepatocyte invasion. ${ }^{19}$

Table 1: Malaria vaccine candidates in preclinical or clinical trials. ${ }^{13,41}$

\begin{tabular}{|c|c|c|c|c|}
\hline $\begin{array}{l}\text { Sr. } \\
\text { No. }\end{array}$ & Malaria vaccine candidate & Antigen (s) used & Target & $\begin{array}{l}\text { Stage of } \\
\text { development }\end{array}$ \\
\hline 1. & RTS,S/ AS01E & $\begin{array}{l}\text { Pf CSP (207-395) \& } \\
\text { HepBsAg }\end{array}$ & PE & Phase-III \\
\hline 2. & GSK RTS,S AS01/AS02 & & PE & Phase II \\
\hline 3. & ChAd63/MVA ME-TRAP & $\begin{array}{l}\text { TRAP+ ME epitopes } 9 \text { CS, } \\
\text { LSA1, LSA3, STARP, EXP1, } \\
\text { pb9) }\end{array}$ & PE & Phase I/IIa \\
\hline 4. & $\begin{array}{l}\text { Adenovirus (Ad 35) vectored } \\
\text { CS \& RTS,S-ASO1 in } \\
\text { heterologous prime- boost } \\
\text { regimen }\end{array}$ & CSP & $\mathrm{PE}$ & Phase I/IIa \\
\hline 5. & $\begin{array}{l}\text { Adenovirus }(\mathrm{Ad} 35) \text { and } \\
\text { adenovirus } 26(\mathrm{Ad} 26) \text { vectored } \\
\text { CS in heterologous prime- } \\
\text { boost regimen }\end{array}$ & CSP & PE & Phase I/IIa \\
\hline 6. & Adenovirus (Ad35) vectured CS & CSP & PE & Phase Ia $\&$ Phase $1 \mathrm{~b}$ \\
\hline 7. & $\begin{array}{l}\text { PfSPZ: metabolically active, } \\
\text { non-replicating (malaria } \\
\text { sporozoites vaccine) }\end{array}$ & $\begin{array}{l}\text { Whole irradiated P. } \\
\text { falciparum sporozoites (Strain } \\
\text { 3D7) }\end{array}$ & PE & Phase I/IIa \\
\hline 8. & PfcelTos FMP012 & CelTOS & PE & \\
\hline 9. & Pf GAP p 52/p32 & $\begin{array}{l}\text { Genetically attenuated } \\
\text { Sporozoites }\end{array}$ & PE & Phase I/IIa \\
\hline 10. & $\begin{array}{l}\text { Adenovirus (Ad26) vectured } \\
\text { CS; Adenovirus (Ad35) } \\
\text { vectured CS }\end{array}$ & CSP & PE & $\begin{array}{l}\text { Phase I with } \\
\text { challenge }\end{array}$ \\
\hline 11. & EBA 175 RII & $\begin{array}{l}\text { EBA } 175 \text { RII, non- } \\
\text { glycosylated }\end{array}$ & BS & Phase 1a, 1b \\
\hline 12. & $\begin{array}{l}\text { FMP2.1/ASO2A ( AMA-I 3D7 } \\
\text { E. coli expressed in ASO2A } \\
\text { adjuvant }\end{array}$ & $\begin{array}{l}\text { AMA-1 (the ectodomain } \\
\text { amino acids } 83 \text { (Gly) to } 531 \\
\text { (Glu)) }\end{array}$ & BS & Phase $1 / 2 \mathrm{a} \& 1 \mathrm{~b}$ \\
\hline 13. & $\begin{array}{l}\text { FMP2.1/ASO2B ( AMA-I 3D7 } \\
\text { E. coli expressed in ASO2B } \\
\text { adjuvant }\end{array}$ & & BS & Phase IIb \& I/IIa \\
\hline 14. & $\begin{array}{l}\text { EMPO10/AS01B (MSP-1 } \\
\text { 42FVO E. coli expressed in } \\
\text { ASOIB adjuvant) }\end{array}$ & MSP-1, p42 subunit & BS & Phase Ia \\
\hline 15. & SE 36 & $\begin{array}{l}\text { N-terminal domain of serine } \\
\text { repeat antigen (SERA5) }\end{array}$ & & \\
\hline 16. & $\begin{array}{l}\text { MVDB/NIAID/NIH AMA1- } \\
\text { C1/ISA720 }\end{array}$ & & BS & Phase I \\
\hline 17. & $\begin{array}{l}\text { WRAIR AMA1 (3D7)/GSK } \\
\text { AS01B/AS02A }\end{array}$ & & BS & Phase I \\
\hline 18. & ChAd63/MVA MSP1 & MSP1 & BS & \\
\hline 19. & ChAd63/ AMA MVA AMA1 & AMA1 & & \\
\hline 20. & ChAd63/ AMA1/MVA AMA1 & AMA1 & BS & Phase I \\
\hline 21. & LaTrobe MSP2-C1/ISA720 & MSP2/ISA 720 & BS & Phase I \\
\hline 22. & MSP[181-276] & MSP3 & BS & \\
\hline 23. & MSP [181-276] field & MSP3 & BS & Phase Ib Pediatric \\
\hline
\end{tabular}




\begin{tabular}{|c|c|c|c|c|}
\hline 24. & ZMZ2 & GLURP, MSP3 & BS & Phase Ia \\
\hline 25. & ZMZ2 field & GLURP, MSP3 & BS & $\begin{array}{l}\text { Phase Ib Gabon } \\
\text { adults }\end{array}$ \\
\hline 26. & Monash MSP4+Monash MSP5 & MSP4 \& MSP5 & BS & Phase 1 \\
\hline 27. & $\begin{array}{l}\text { GenVec's multi-antigen vaccine } \\
\text { (Adenovirus serotype-5 vector) }\end{array}$ & $\begin{array}{l}\text { CSP:Ag2:LSA-1+ } \\
\text { GenVec Ad5-AMA1:MSP1 }\end{array}$ & $\mathrm{PE}+\mathrm{BS}$ & Preclin \\
\hline 28. & JAIVAC (MSP1 19/EBA175) & MSP1, 19/EBA175 & BS & Phase I \\
\hline 29. & $\begin{array}{l}\text { AMA1-C1/Alhydrogel+CPG } \\
7909\end{array}$ & $\begin{array}{l}\text { AMA1-C1: mixture of two } \\
\text { AMA1 proteins, AMA1-FVO } \\
\text { and AMA1 }\end{array}$ & BS & Phase Ia \& I/IIa \\
\hline 30. & $\begin{array}{l}\text { BSAM-2/Alhydrogel }{ }^{\circledR}+\mathrm{CPG} \\
7909\end{array}$ & $\begin{array}{l}\text { BSAM-2 is a mixture of } \\
\text { MSP1 42-FVO, MSP1 42- } \\
\text { 3D7 and two AMA1 }\end{array}$ & BS & Phase Ia \& Ib \\
\hline 31. & ICGEB- PvRII/AS01B & Based on DBP & $\begin{array}{l}\text { P. vivax } \\
\text { (Whole } \\
\text { parasite) }\end{array}$ & Preclinical \\
\hline 32. & VMP001/AS01B & CSP & P. vivax & \\
\hline 33. & NMRC-M3V-Ad-PfCA & $\begin{array}{l}\text { PfCSP (3D7 strain) in } \\
\text { combination with PfAMA1 } \\
\text { (3D7 strain) }\end{array}$ & & Phase I/IIa \\
\hline 34. & $\begin{array}{l}\text { NMRC-M3V-D/Ad- PfCA } \\
\text { Prime/Boost }\end{array}$ & $\begin{array}{l}\text { PfCSP (3D7 strain) in } \\
\text { combination with PfAMA1 } \\
\text { (3D7 strain) }\end{array}$ & $\mathrm{PE}+\mathrm{BS}$ & Phase I/IIa \\
\hline 35. & $\begin{array}{l}\text { CSP, AMA1 virosomes (PEV } \\
301,302) \text {. }\end{array}$ & AMA-1 and CS mimotopes. & $\mathrm{BS}$ & $1 \mathrm{a}, 1 \mathrm{~b}$ \\
\hline 36. & Pfs25-EPA & Pfs25-EPA & $\begin{array}{l}\text { Sexual } \\
\text { stage }\end{array}$ & Phase I \\
\hline 37. & Plasmodium vivax CSP & P. vivax project & & \\
\hline
\end{tabular}

GSK= GlaxoSmithKline Biologicals; WRAIR=US Walter Reed Army Institute of Research; MVDB= Malaria Vaccine Development Branch; NIAID= US National Institute of Allergy and Infectious Diseases; NMRC=Naval Medical Research Center (US Military Malaria Vaccine Program) (USA); ICGEB= The International Center for Genetic Engineering and Biotechnology, New Delhi; PATH=Program for Appropriate Technology in Health, MVI= The PATH Malaria Vaccine Initiative; $\mathrm{DBP}=$ Duffy binding protein; $\mathrm{ME}-\mathrm{TRAP}=$ Multiple epitome -thrombospondin related adhesive protein; MVA=Modified vaccinia virus Ankara; HepBsAg= Hepatitis B surface antigen; CelTOS $=$ Cell traversal protein for ookinetes and sporozoites; AMA1=Apical membrane antigen 1; FVO= Falciparum Vivax Ovale.

3. Multi-antigen, multi-stage (PE +BS) vaccines incorporating CSP: PE+BS vaccines incorporate BS antigens that target attachment or invasion mechanisms of merzoites, and erythrocyte-binding antigen and reticulocyte-binding protein are leading candidates. ${ }^{19}$

4. Whole sporozoite-based PE vaccines: Radiationattenuated sporozoites are potential vaccines for $P$. falciparum malaria. Radiation-attenuated sporozoites delivered by mosquito bite have induced very high levels of protection, more than $90 \%$, though number of volunteers in the trials was very small. ${ }^{25}$

A Phase $1 / 2$ a clinical study designed to determine the protective efficacy of whole radiation-attenuated sporozoites, delivered for the first time by needle and syringe, was initiated in $2009 .{ }^{19}$

\section{P. VIVAX VACCINE}

Two leading P. vivax antigens, CSP and RII domain of the Duffy binding protein (DBP), are in clinical testing in human challenge studies and are most promising vaccine candidates for P. Vivax. ${ }^{19}$

\section{MULTI-ANTIGEN/MULTI-STAGE PE + BS}

The combination of CSP and DBP based vaccines, have a reasonable expectation for synergy, and will depend on the outcome of challenge studies for the two individual antigens. $^{14}$

\section{TRANSMISSION-BLOCKING VACCINES (TBVs)}

Blockade of cycle of transmission of Plasmodium has a key role in the global control, elimination and eradication effort of malaria. Most effective TBV for malaria is PE vaccine that not only blocks cycle of transmission in human host but also protects from clinical disease. ${ }^{19}$

\section{SEXUAL-STAGE VACCINES}


Fertilization can be prevented in the mosquito and its blood meal by induction of antibodies to gametocyte antigens; thus assessment of the efficacy of gametocyte vaccines is possible with a simple ex-vivo assay. Sexual stage vaccination would not protect the vaccinated individuals from malaria but would protect communities from infection. P. falciparum gametocyte candidate vaccine, Pfs25, a recombinant protein is under development by US NIAID Malaria vaccine development unit. $^{26}$

\section{BLOOD-STAGE VACCINES}

A vaccine that could prevent invasion of red blood cells by merozoites (anti-invasion) would prevent clinical attacks of malaria. Merozoite surface protein-1 (MSP-1) is most well characterized antigen involved in invasion and is the basis for several candidate vaccines. Parallel pathways of invasion and demonstration of antibodies to MSP-1 blocking malaria-protective antibodies have complicated vaccine development. ${ }^{27}$ An anti-invasion vaccine based on MSP-1, known as falciparum malaria protein (FMP-1), is progressing for an adult phase-I study in western Kenya. ${ }^{26}$

A recombinant viral vaccine, NYVAC Pf-7 (P falciparum- 7), has been developed that encodes seven antigens from various life-cycle stages. ${ }^{28}$ Results of a sporozoite challenge study of NYVAC Pf-7 showed encouraging delays in time to parasitaemia, and some antibody and cytotoxic T-lymphocyte immunogenicity, but this candidate has not been further developed. Two blood-stage candidates, glutamate rich protein (GLURP) and MSP3, have been clinically assessed in Europe. ${ }^{29-30}$ With all such protein candidates key issue is identification of a safe, immunogenic adjuvant, since the traditional adjuvant, alum seems to be insufficiently immunogenic for many malaria proteins. Also vaccines with an alum adjuvant induce a Th2 response, rather than the generally more desirable Th1 response. Induction of biologicallyrelevant antibodies is a further challenge, because these may require a native conformation of the recombinant protein. $^{26}$

\section{VACCINE DEVELOPMENT IN THE POST- GENOMIC ERA}

Whole-genome sequencing indicates that there are probably $5300 \mathrm{P}$. falciparum antigens and the genome databases can be used for identifying hundreds of the vaccine candidates. Identification of antigens does not help solve some key problems in malaria vaccine development: how to induce strong, durable immune responses; and how to combine multiple antigens without interference or competition. ${ }^{26}$

\section{MALARIA VACCINE EVALUATION TECHNOLOGIES}

Various evaluation technologies are employed for effective preclinical and clinical evaluation of candidate malaria vaccine, including:

Human challenge studies: This model has been used effectively in the clinical development of RTS,S particularly to identify optimal adjuvant formulations and immunization regimens.

CMI-based techniques: Novel CMI-based techniques are used to better evaluate the quality of immune responses, in both preclinical and clinical candidate studies.

Preclinical studies: Preclinical studies are used to support comparative immunogenicity and efficacy studies that are critical in development decisions. A range of different systems, including the P. knowlesi non-human primate challenge model and chimeric rodent and nonhuman primate parasites expressing vaccine target genes from $\mathrm{P}$. falciparum, are being evaluated to facilitate testing of $P$. falciparum vaccine concepts in preclinical models.

Functional assays: To address the increased focus on the development of P. vivax vaccine and TBVs, in several key areas evaluation technologies need to be applied. The relapse infections caused by liver-stage hypnozoites not seen in P. falciparum and absence of culture system for P. vivax hypnozoites, are important considerations. An effective functional assay to support the preclinical and clinical development of TBVs requires standardization and less complex assays with higher throughput capacity (e.g., ELISA surrogate) must be developed to effectively support preclinical and clinical studies of TBVs. ${ }^{19}$

\section{SAFETY, EFFICACY AND COST CONSIDERATIONS}

A partial-efficacy vaccine can have a significant impact e.g. a blood-stage vaccine with efficacy of 50 percent against severe disease and duration of efficacy of at least one year can have a significant impact on the malaria burden. This vaccine could prevent an estimated 153 deaths per 100,000 infants vaccinated in Africa and save 4,910 DALYs per 100,000 in the same target group. ${ }^{31}$ Genton and Corradin suggest that a malaria vaccine with greater than $50 \%$ efficacy might be highly cost-effective for endemic areas. ${ }^{32}$ Vaccine efficacy and efficacy of RTS,S/AS02A candidate malaria vaccine has been demonstrated by various clinical trials. ${ }^{33-37}$ RTS,S/AS01 is now in very large phase III efficacy trials enrolling 15000 children in sites in 7 African countries with aim of licensure and deployment in 2015, an encouraging process towards $1^{\text {st }}$ generation malaria vaccine. ${ }^{38}$ A lead candidate, malaria vaccine, RTS/AS01E has shown efficacy against falciparum malaria in 5-17 month old children in a randomized controlled trial. ${ }^{39}$ In a study antibody and cellular responses elicited by RTS,S lasted for 6 months during follow- up; and efficacy of the vaccine was variably linked to antigen, adjuvants used and dose. $^{40}$ A high-efficacy vaccine (efficacy of 90 
percent against clinical and severe disease, with minimal efficacy decay) could have a tremendous impact and a widespread acceptance by countries, and improved EPI coverage rates; thus could be a powerful tool for reducing the malaria burden. A highly efficacious, long-duration vaccine could have an estimated average cost effectiveness in Africa of approximately \$14 per DALY saved and $\$ 508$ per death averted. The impact of partial and high efficacy vaccines will vary from country to country, thus even a partial efficacy vaccine will be supplemental to the existing and future interventions in malaria control and the immunization programs. Both types of vaccine are cost-effective and significantly reduce the burden of malaria for many African countries. In case of partial-efficacy vaccine, it is important to note that for at risk populations other methods (e.g., bed nets, long-sleeved clothing) will remain particularly important, even for individuals who have been immunized. An early action is important for the successful malaria vaccine introduction in the future. Although immunization coverage rates have improved dramatically over the past few decades, there is still a room for progress. Improving the reach and capacity of immunization programs will increase the future impact of malaria vaccines, as well as other immunizations. ${ }^{31}$

\section{SUMMARY}

Development of an effective and safe malaria vaccine is a technical possibility in the view of malaria researchers and the one is urgently needed. Efficacy studies with malaria vaccine have to progress through adults and children aged 1-5 years before reaching their target age group of infants. There will probably be a need for combination vaccines, and therefore vaccine development efforts of several groups will almost certainly have to be combined. Although one candidate vaccine has moved from first use in human participants to a phase-I trial in developing countries within months, a greater challenge is speeding the progression from demonstrated efficacy to licensing of a vaccine. The cost of vaccines should be considered before large scale efficacy trials are planned. Estimation of cost is complicated by the unpredictable, but decrease in price of vaccine overtime can be anticipated. The establishment of a global purchase fund could be essential to spur industrial interest in late-stage vaccine development.

\section{REFERENCES}

1. Moree M, Ewart S. Policy challenges in malaria vaccine introduction. Am J Trop Med Hyg 2004 Aug; 71(Suppl 2):248-52.

2. WHO. WHO Global Malaria Programme: The World Malaria Report 2010. World health Organization 2010. Available at http://whqlibdoc.who.int/publications/2010/9789241 564106_eng.pdf. Accessed 19 August 2012.
3. Ie Sueur D, Sharp BL, Gouws E, Ngoxongo S. Malaria in South Africa. S Afr Med J 1996;86:9369.

4. WHO. World Health Report: Reducing Risks, Promoting Healthy Life. Geneva: World Health Organization 2002.

5. Aide P, Bassat Q, Alonso PL. Towards an effective malaria vaccine. Arch Dis Child 2007;92(6):476-9.

6. Bonn D. Filling the vaccine gap. Lancet Infect Dis 2005;5(1):7.

7. Gupta S, Snow RW, Donnelly CA, et al. Immunity to non-cerebral severe malaria is acquired after one or two infections. Nat Med 1999;5(3):340-3.

8. Rieckmann KH, Beaudoin RL, Causells JS, et al. Use of attenuated sporozoites in the immunization of human volunteers against falciparum malaria. Bull World Health Organ 1979:57(Suppl 1):261-5.

9. Alonso PL, Sacarlal J, Aponte JJ, et al. Efficacy of RTS,S/AS02A vaccine against plasmodium falciparum infection and disease in young African children: randomized controlled trial. Lancet 2004;364(9443):1411-20.

10. Alonso PL, Smith T, Schellenberg JR, et al. Randomized trial of efficacy of SPf66 vaccine against plasmodium falciparum malaria in children in southern Tanzania. Lancet 1994;344(8931):117581.

11. Engers HD, Godal T. Malaria vaccine development: current status. Parasitol Today 1998;14:56-64.

12. Beverley PC. Immunology of vaccination. Br Med Bull 2002;62:15-28.

13. Malaria Vaccine Technology Roadmap. Available at http://www.malariavaccine.org/malvacroadmap.php. Accessed 2 March 2012.

14. McGregor IA. Mechanism of acquired immunity and epidemiological patterns of antibody responses in malaria in man. Bull World Health Organization 1974;50:259-66.

15. Cohen S, McGregor IA, Carrington S. Gamma globulin and acquired immunity to malaria. Nature 1961;192:733-7.

16. Marsh K, Howard RJ. Antigens induced on erythrocytes by P. falciparum: expression of diverse and conserved determinants. Science 1986;231:1503.

17. Bouharoun-Tayoun H, Attanath P, Sabchareon A, Chongsuphajaisiddhi T, Druilhe P. Antibodies that protect humans against Plasmodium falciparum blood stages do not on their own inhibit parasite growth and invasion in vitro, but act in cooperation with mosquitos. J Exp Med 1990;172:1633-41.

18. Doolan DL, Hoffman SL. DNA-based vaccines against malaria: status and promise of the MultiStage Malaria DNA vaccine operations. Int $\mathrm{J}$ Parasitol 2001;31:753-62.

19. PATH malaria vaccine initiative (MVI). Available at http://www.malariavaccine.org/malvacapproaches.php. Accessed 2 March 2012.

20. Stoute JA, Slaoui M, Heppner DG, et al. A preliminary evaluation of a recombinant 
circumsporozoite protein vaccine against $\mathrm{P}$. falciparum malaria. RTS,S Malaria vaccine evaluation group. N Engl J Med 1997;336(2):86-91.

21. Potocnjak P, Yoshida N, Nussenzweig RS, Nussenzweig V. Monovalent fragments (Fab) of monoclonal antibodies to a sporozoites surface antigen $(\mathrm{Pb} 44)$ protect mice against malarial infection. J Exp Med 1980;151:1504-13.

22. Nardin EH, Nussenzweig V, Nussenzweig RS, et al. Circumsporozoite proteins of human malarial parasites Plasmodium falciparum and Plasmodium vivax. J Exp Med 1982;156:20-30.

23. Hill AV. Vaccines against malaria. Philos Trans R Soc Lond B Biol Sci 2011;366:2806-14.

24. Birkett A, Lyons K, Schmidt A, et al. A modified hepatitis $\mathrm{b}$ virus core particle containing multiple epitomes of the P. falciparum CSP protein provides a highly immunogenic malaria vaccine in preclinical analysis in rodent and primate hosts. Infect Immun 2002;70:6860-70.

25. Hoffman SL, Goh LM, Luke TC, Schneider I, et al. Protection of humans against malaria by immunization with radiation-attenuated Plasmodium falciparum sporozoites. J Infect Dis 2002;185:115564.

26. Moorthy VS, Good MF, Hill AV. Malaria vaccine developments. Lancet 2004;363:150-6.

27. Holder AA, Guevara Patino JA, Uthaipibull C, et al. Merozoite surface protein 1 , immune evasion, and vaccines against sexual blood stage malaria. Parassitologia 1999;41:409-14.

28. Ockenhouse CF, Sun PF, Lanar DE, et al. Phase I/IIa safety, immunogenicity, and efficacy trial of NYVAC-Pf7, a pox-vectored, multiantigen, multistage vaccine candidate for $P$. falciparum malaria. J Infect Dis 1998;177:1664-73.

29. Oeuvray C, Theisen M, Rogier C, Trape JF, Druilhe P. Cytophilic immunoglobulin responses to plasmodium falciparum glutamate rich protein are correlated with protection against clinical malaria in Dielmo, Senegal. J Infect Immun 2000;68:2617-20.

30. Oeuvray C, Bouharoun-Tayoun H, Gras-Masse H, et al. Merozoite surface protein-3: a malaria protein inducing antibodies that promote $\mathrm{P}$. falciparum killing by cooperation with blood monocytes. Blood 1994;84(5):1594-602.

31. Briefing Document: National decision-making framework for malaria vaccines. Available at http://malvacdecision.net/wp-
content/uploads/2012/03/D-MakingBrief-ImpactFinal.pdf. Accessed 10 March 2012.

32. Genton B, Corradin B. Malaria vaccines: from laboratory to the field. Curr Drug Targets Immune Endocr Metabol Disord 2002;2:255-67.

33. Abdulla S, Oberholzer R, Juma O, et al. Safety and immunogenicity of RTS,S/AS02D malaria vaccine in infants. N Engl J Med 2008;359:2533-44.

34. Alonso PL, Sacarlal J, Aponte JJ, et al. Duration of protection with RTS,S/AS02A malaria vaccine in prevention of Plasmodium falciparum disease in Mozambican children: single-blind extended followup of a randomised controlled trial. Lancet 2005;366(9502):2012-18.

35. Aponte JJ, Aide P, Renom M, et al. Safety of the RTS,S/AS02D candidate malaria vaccine in infants living in a highly endemic area of Mozambique: a double blind randomised controlled phase I/IIb trial. Lancet 2007;370(9598):1543-51.

36. Bejon P, Lusingu J, Olotu A, et al. Efficacy of RTS,S/AS01E vaccine against malaria in children 5 to 17 months of age. N Engl J Med 2008;359:252132.

37. Sacarlal J, Aide P, Aponte JJ, Renom M, Leach A, Mandomando I, et al. Long-term safety and efficacy of RTS,S/AS02A malaria vaccine in Mozambican children. J Infect Dis 2009;200:329-36.

38. Guinovart C, Aponte JJ, Sacarlal J, Aide P, Leach A, Bassat $\mathrm{Q}$, et al. Insights into log-lasting protection induced by RTS,S/AS02A malaria vaccine: further results from a phase IIb trial in Mozambican children. PLoS One. 2009;4(4):e5165.

39. Olotu A, Lusingu J, Leach A, Lievens M, Vekemans J, Msham S, et al. Efficacy of RTS,S/AS01E malaria vaccine and exploratory analysis on anticircumsporozoite antibody titres and protection in children aged 5-17 months in Kenya and Tanzania: a randomised controlled trial. Lancet Infect Dis 2011;11(2):102-9.

40. Stoute JA, Kester KE, Krzych U, Wellde BT, Hall $\mathrm{T}$, White $\mathrm{K}$, et al. Long-term efficacy and immune responses following immunization with the RTS,S malaria vaccine. J Infect Dis 1998:178:1139-44.

41. WHO. Initiative for Vaccine Research (IVR) Available http://www.who.int/vaccine_research/links/Rainbow /en/index.html. Accessed 11 September 2012. 